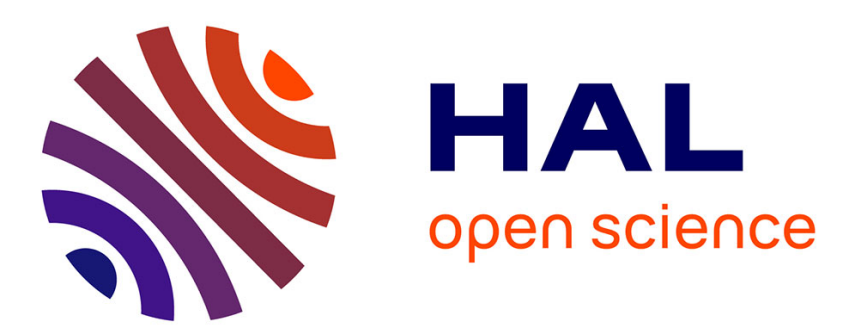

\title{
Interstitial Orbitals for Simple Metals: 2D Systems
}

Isabelle Baraille, Marie-Bernadette Lepetit

\section{To cite this version:}

Isabelle Baraille, Marie-Bernadette Lepetit. Interstitial Orbitals for Simple Metals: 2D Systems. The Journal of physical chemistry, 1996, 100 (10), pp.4224-4228. 10.1021/JP953119G . hal-03225267

\section{HAL Id: hal-03225267 \\ https://hal-univ-pau.archives-ouvertes.fr/hal-03225267}

Submitted on 12 May 2021

HAL is a multi-disciplinary open access archive for the deposit and dissemination of scientific research documents, whether they are published or not. The documents may come from teaching and research institutions in France or abroad, or from public or private research centers.
L'archive ouverte pluridisciplinaire HAL, est destinée au dépôt et à la diffusion de documents scientifiques de niveau recherche, publiés ou non, émanant des établissements d'enseignement et de recherche français ou étrangers, des laboratoires publics ou privés. 


\title{
Interstitial Orbitals for Simple Metals: 2D Systems
}

\author{
Isabelle Baraille \\ Laboratoire de Chimie Structurale, IFR, rue Jules Ferry, 64000 Pau, France
}

\author{
Marie-Bernadette Lepetit* \\ Laboratorie de Physique Quantique, IRSAMC, 118 route de Narbonne, 31062 Toulouse Cedex, France
}

Received: October 23, $1995^{\otimes}$

The relevance of the interstitial model for the metallic bond has been studied on 2D simple metal lattices: the lithium square lattice and the magnesium triangular one. As on 1D systems the valence electron density is essentially interstitial on both systems. However, the magnetic treatment of the inverse lattice problem-up to now always associated with the interstitial description of simple metals-was proven false on the magnesium lattice which remains highly delocalized. The reasons of the different behavior of the $\mathrm{Li}$ and $\mathrm{Mg}$ systems have been investigated, as well as the conductivity problem associated with a magnetic description of the inverse lattice. A phase transition, semiconductor versus insulator, has been exhibited on the $\mathrm{Mg}$ lattice. The insulator phase (long distances) corresponding to an atom-centered solution, the semiconductor phase (short distances) corresponding to an interstitial solution.

\section{Introduction}

In the building of a covalent bond, the valence electron density increases between the two atoms, but the density maxima stay on the atoms. The $\mathrm{H}_{2}$ molecule, for instance, is well described from the s orbitals of the hydrogen atoms. In a simple metal bond however, the valence density maximum is shifted from the atoms to the middle of the bond. The $\mathrm{Li}_{2}$ molecule, for example, exhibits such a behavior. In this case the s orbitals of the atoms are not sufficient to build a reasonable first-order approximation of the diatom wave function, the contribution of the $\mathrm{p}_{\sigma}$ orbitals being too important. The same type of behavior has been witnessed in metallic clusters, ${ }^{1-3}$ the density maxima being located in the interstices between the atoms, and the $s-p$ hybridization being very strong. From the bulk point of view, solid-state physicists would describe the building of such a bond by the overlapping of the lithium $\mathrm{s}$ and $\mathrm{p}$ bands, the valence electrons moving freely in the resulting hybridized $\mathrm{s}-\mathrm{p}$ band, which spans the interstices between the atoms.

From this analysis, the idea of describing the simple metals in terms of interstitial orbitals comes quite naturally. The initial interstitial model (proposed by McAdon and Goddard ${ }^{2}$ ) involved three different ideas: (1) the valence space is spanned by interstitial orbitals (IO) localized between the atoms; (2) the $\alpha / \beta$ coupling is abandoned, the IO being singly occupied; (3) when building the metallic bonds, i.e., going from an atomic localization of the valence electrons to an interstitial one, one goes also from a strongly delocalized picture to a strongly correlated one (the valence configuration interaction (CI) being well reproduced by a magnetic Hamiltonian); this last idea was stressed by one of us (M.B.L.) and co-workers. ${ }^{4}$

This model has been confirmed on clusters ${ }^{4}$ where its results (for energies as well as for wave functions) were successfully compared to ab initio valence complete active space selfconsistent field (CASSCF) calculations. The same model was proved relevant on $1 \mathrm{D}$ systems $^{5}$ (lithium chain).

One may however wonder whether the interstitiality of the valence density (point 1) and the strong correlation hypothesis (points 2 and 3) are indissociable.

\footnotetext{
${ }^{\otimes}$ Abstract published in Advance ACS Abstracts, February 1, 1996.
}

TABLE 1: Basis Sets

\begin{tabular}{|c|c|c|c|c|c|c|}
\hline & \multicolumn{3}{|c|}{ lithium } & \multicolumn{3}{|c|}{ magnesium } \\
\hline & \multicolumn{2}{|c|}{ atomic } & \multirow{2}{*}{$\begin{array}{l}\text { interstitial } \\
\quad \exp \end{array}$} & \multicolumn{2}{|c|}{ atomic } & \multirow{2}{*}{$\begin{array}{l}\text { interstitial } \\
\text { exp }\end{array}$} \\
\hline & $\exp$ & coef & & $\exp$ & coef & \\
\hline \multirow[t]{4}{*}{$\mathrm{s}$} & 2.4642 & -0.0164 & 0.09 & 0.4342 & -0.0135 & 0.14 \\
\hline & 1.9914 & 0.0358 & & 0.7570 & -0.1350 & \\
\hline & 0.5819 & 0.1321 & & 0.1010 & 0.6422 & \\
\hline & 0.0709 & -0.6071 & & & & \\
\hline$s^{\prime}$ & 0.0600 & 1.0000 & & 0.0700 & 1.0000 & \\
\hline $\mathrm{p}$ & 0.1200 & 1.0000 & & 0.0950 & 1.0000 & \\
\hline
\end{tabular}

The present work will study the application of the above model to 2D systems, a square lithium lattice and a triangular magnesium one. We shall see that while the lithium slab follows well all the hypotheses of the original model, the magnesium system is well described in terms of IO but remains a highly delocalized system. We shall discuss the possible reasons of this difference.

\section{Results}

The calculations performed in this work have been made using the unrestricted Hartree-Fock (UHF) ${ }^{6}$ version of the $a b$ initio Hartree-Fock CRYSTAL program. ${ }^{7}$ The calculations were performed using a double cell (four atoms, four squares for the lithium; four atoms, eight triangles for the magnesium) and the $P 1$ space symmetry group -in order to allow symmetry breakings to occur.

Atomic basis sets (see Table 1) are of valence double- $\zeta$ plus polarization quality, the core electrons are treated using a pseudopotential method. ${ }^{8}$ To allow more flexibility and to avoid higher 1 atomic wave functions, we also used an intersticecentered s orbital (located at the center of the square for the lithium system, and at the center of the triangle for the magnesium one). Their exponents were optimized so that to minimize the energy of the lowest HF solution.

All energies given in this paper do take into account corrections for basis set superposition errors; for each geometry, the energy of the free atom is given by a calculation of one atom, surrounded by ghost atoms which support the appropriate basis set. These ghosts are located at the positions where the neighboring atoms or interstices would be in the $2 \mathrm{D}$ system. 


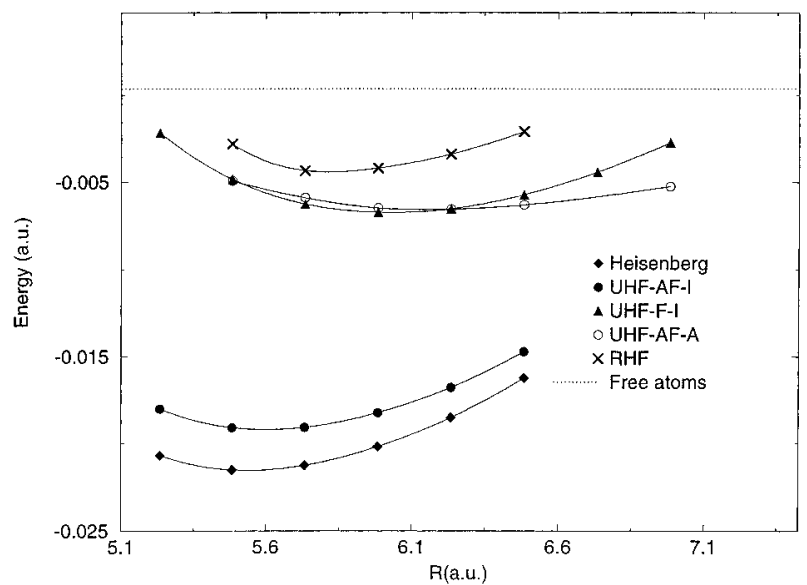

Figure 1. Lithium square lattice: potential curves of the different HF solutions.

A. Lithium Square Lattice. Restricted Hartree-Fock (RHF) calculations as well as different UHF calculations have been performed on this system.

Figure 1 reports the different potential curves.

As for the lithium clusters, but contrarily to the 1D chain, the RHF solution is weakly bounded ( $E_{\text {coh/at }}=0.129 \mathrm{eV}$ at $R_{\text {eq }}$ $=3.105 \AA)$. The analysis of the Mulliken population exhibits an essentially atomic s population ( $\geq 75 \%$ at $5.75 \mathrm{au})$ associated to a nonnegligible $p_{\sigma}$ contribution $(0.174$ at $5.75 \mathrm{au})$, the interstice-centered s orbitals are however only weakly populated ( 0.057 at $5.75 \mathrm{au})$. Let us remember that this solution is not asymptotically correct, since it dissociates in a mixture of neutral and ionic atomic states.

As can be seen in Figure 1, the lowest solution is the UHF antiferromagnetic interstitial solution (UHF-AF-I). This solution is strongly bounded, its cohesive energy is $E_{\text {coh/at }}=0.532 \mathrm{eV}$ at the interactomic distance of $R_{\mathrm{eq}}=2.98 \AA$. The study of both the Mulliken population analysis and the valence charge density map (see Figure 2a) shows its interstitial character. The valence density maxima are located at the center of the squares. They constitute an inverse square lattice isomorphic to the original one (1 square for 1 atom and 1 electron). The population of the atomic s orbitals is only about $50 \%$ of the total one, the $p_{\sigma}$ orbitals (with for instance $0.226 \bar{e}$ at $5.75 \mathrm{au}$ ) and the interstitice-centered s orbitals (with $0.266 \bar{e}$ at $5.75 \mathrm{au}$ ) share the remaining $50 \%$. The spin density map (see Figure $2 b)$ as well as the $\alpha-\beta$ population analysis exhibit a large spin density wave on the interstices, at 5.75 au:

$$
\frac{\rho_{\alpha}-\rho_{\beta}}{\rho_{\alpha}+\rho_{\beta}}=0.88
$$

As in the lithium systems studied in refs 4 and 5, the corresponding interstitial ferromagnetic solution (UHF-F-I) was found to be stable. Its cohesive energy is $E_{\text {coh/at }}=0.194 \mathrm{eV}$ (i.e., larger than the binding energy of the RHF solution), and its equilibrium geometry is slightly larger than the antiferromagnetic one $\left(R_{\mathrm{e} 1}=3.197 \AA\right)$. Its interstitial character is undoubtable, the charge density presenting large maxima at the square centers, the Mulliken population analysis exhibiting an even larger contribution of both the $p_{\sigma}(0.240$ at $5.75 \mathrm{au})$ and the interstitice-centered s orbitals $(0.356 \bar{e}$ at $5.75 \mathrm{au})$. The stability of those ferromagnetic solutions is clearly due to the strong electronic delocalization in the interstices that lowers the kinetic energy with respect to the ferromagnetic atomic solutions, which are always repulsive. We did not perform a localization of the Block orbitals in order to obtain the interstitial
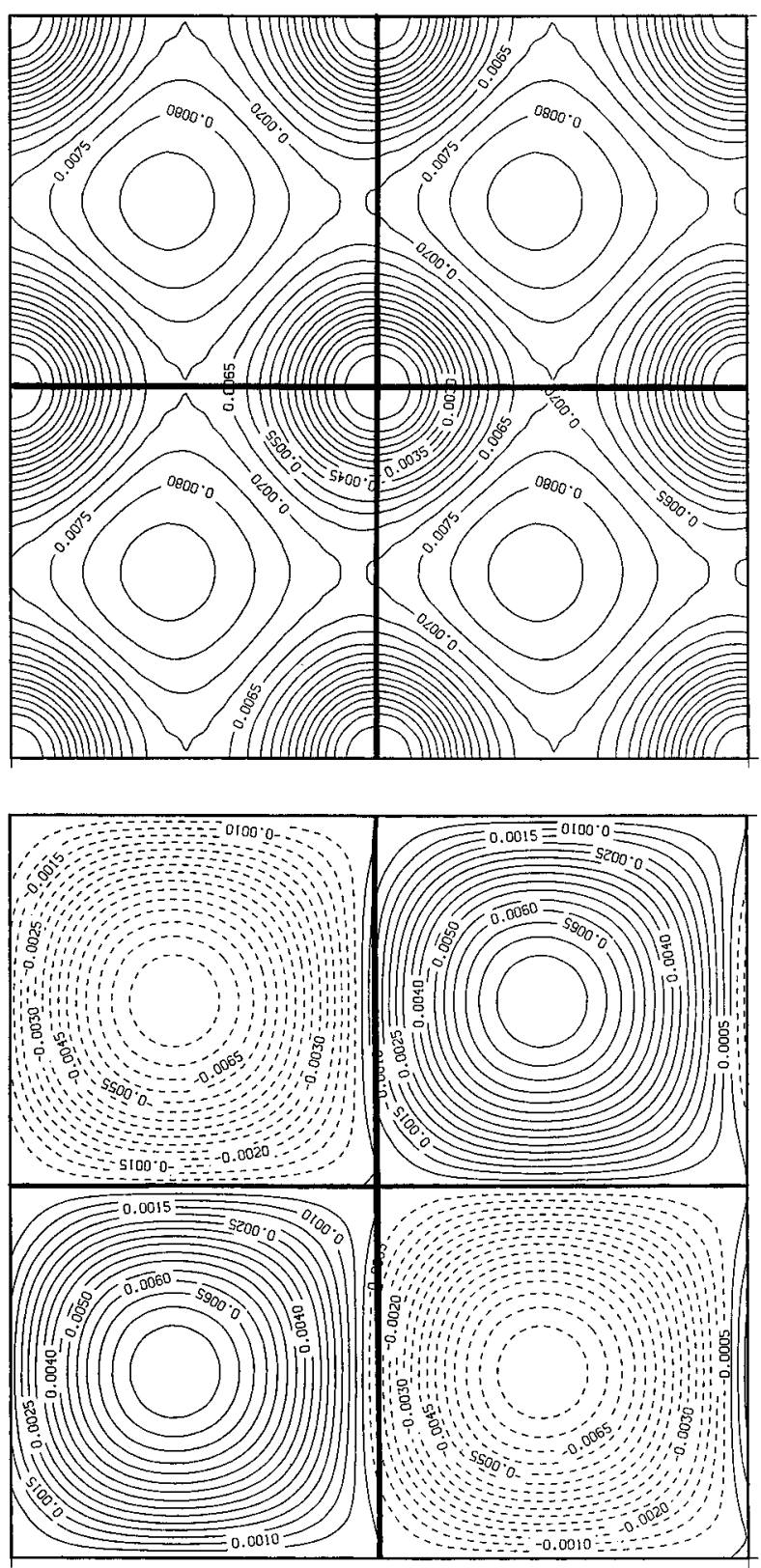

Figure 2. Antiferromagnetic interstitial solution $(6 \AA)$. The nuclei are located on the lattice vertices. (a, top) Valence charge density map. (b, bottom) Valence spin density map. Positive spin, solid lines; negative spins, dashed lines.

Wannier orbitals; however, the stability of the UHF ferromagnetic solution, the strong localization of the valence density in the interstices, the one-to-one correspondence between density maxima and electrons shows us that there is a set of Wannier orbitals strongly localized in the interstices with small orthogonalization tails. It is obvious that the same remarks can be done for the intertitial antiferromagnetic solution, with the same conclusions, i.e., the $\alpha$ and $\beta$ Wannier orbitals would be strongly localized in neighboring interstices with only small delocalization tails.

In the Hartree-Fock approximation, the UHF atomic solution is the correct dissociation limit. Such a solution (the ground state at long interatomic distances) does also exist at short interatomic ones. The antiferromagnetic atomic UHF solution (UHF-AF-A) was computed. It does coexist with the interstitial antiferromagnetic UHF solution. As can be expected, this solution is weakly bounded ( $E_{\text {coh/at }}=0.188 \mathrm{eV}$ at $R_{\text {eq }}=3.275$ $\AA$ ). The Mulliken population analysis confirms the atomic character of this solution with a population of an essentially 


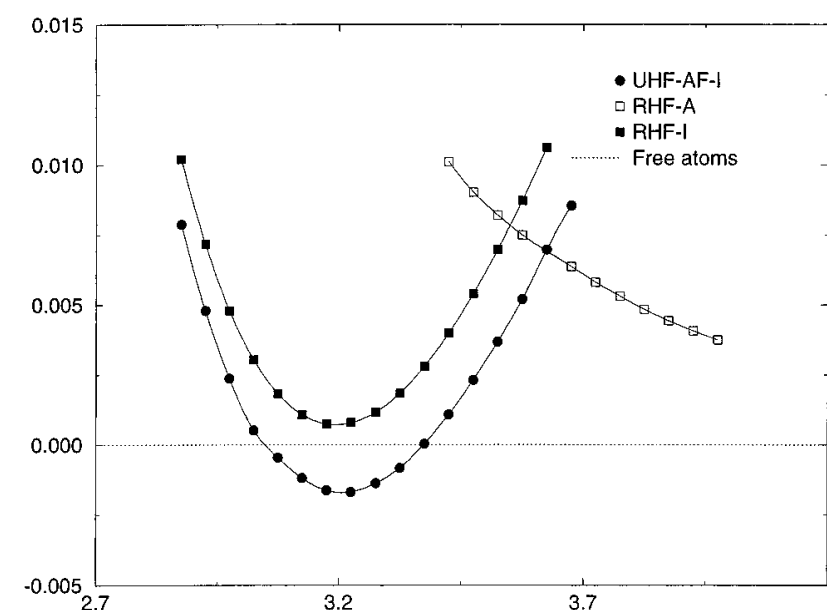

Figure 3. Magnesium triangular lattice. Potential curves of the different HF solutions.

atomic s character $(0.829$ at $5.75 \mathrm{au})$; the contribution of the $p_{\sigma}$ orbitals is much smaller than in the interstitial solution $(0.172$ at $5.75 \mathrm{au}$ ), decreasing quickly when the interatomic distance increases, i.e., equivalent to the $p_{\sigma}$ population of the RHF solution). The population of the square-centered $\mathrm{s}$ orbitals is negligible (0.001 at $5.75 \mathrm{au})$.

The strong localization of the valence electrons in the interstices for the UHF-AF-I solution, the large spin-density wave associated, and the existence of a bounded ferromagnetic interstitial solution show that, as for the clusters and the chain, the square lithium lattice is, in its inverse picture, a highly correlated system, well described by a magnetic approach. We extracted the $J$ parameter of a Heisenberg Hamiltonian as a function of the interatomic separation. Following the Anderson approach, ${ }^{9}$ the UHF-AF-I solution can be assimilated to a Neel state; therefore, the energy difference between the interstitial ferromagnetic and antiferromagnetic UHF solutions is equal to the Heisenberg $J$ parameter (for further details and justifications about the procedure see ref 5). The ground state of this magnetic Hamiltonian corresponds to the valence correlated wave function, its energy is known to be $E_{\text {ferro }}-1.1692 \mathrm{~J} .{ }^{10}$ It is bounded by $E_{\text {coh/at }}=0.595 \mathrm{eV}$ and its equilibrium distance is $R_{\mathrm{eq}}=2.946$ $\AA$.

From this study, one can conclude that the lithium square lattice, as the lithium clusters and 1D chain follows well the interstitial/magnetic model, i.e., (i) there is a shift of the valence electron density from the atoms to the interstices (here the squares) which constitute an inverse lattice and (ii) there is an associated shift from a highly delocalized system in the original atomic lattice to a highly correlated one in the (interstitial) inverse lattice description.

B. Magnesium Triangular Lattice. For the magnesium triangular lattice, we obtained two different RHF solutions (an atom-centered one and an interstice-centered one) and a UHF interstitial antiferromagnetic solution.

Figure 3 displays the potential curves of the different solutions.

The magnesium atom is a closed-shell system and the $\mathrm{Mg}_{2}$ molecule is a van der Waals system; therefore one could naively expect that the lowest HF solution would be an atom-centered solution, but such a description, corresponding to a filled s-band system, could not account for the $\mathrm{Mg}$ metallic nature. The calculations on the triangular magnesium lattice exhibit two different RHF solutions. Both have the same $P 3$ symmetry and coexist as two true minima of the potential RHF surface over a whole range of interatomic distances (from 3.45 to $3.65 \AA$ ). What are those solutions? At short interatomic distances, the most stable solution (RHF-I) presents an interstitial character, while the other solution (RHF-A) has an atomic one. Their energetic order is reversed at long interatomic distances, the atomic solution being the lowest one, as can be expected from the dissociation limit in closed-shell $\mathrm{Mg}$ atoms. The crossing point is at $3.58 \AA$ (see Figure 3). From those simple observations, one can say that the RHF potential energy surface presents two nonintersecting valleys, i.e., a bistability phenomenon.

Both the valence density map (Figure 4a) and the Mulliken population analysis show the interstitial character of the RHF-I solution. As one sees in Figure 4a, the density maxima are located at the triangle centers. Let us note that the associated inverse lattice is isomorphic to the (001) graphite plane. The number of triangles is equal to the number of electrons, leading to a half-filled band in this inverse lattice picture. Unlike the lithium system, the density maxima are very small; the electron density remains strongly delocalized, evenly spread on the bonds of the inverse lattice- as are the $\pi$ electrons in the graphite slab. On the other hand, the Mulliken population analysis exhibits a strong $\mathrm{s}-\mathrm{p}$ mixing with less than $50 \%$ of the population on the $\mathrm{s} \mathrm{Mg}$ orbitals, the remaining population being on the $\mathrm{p}_{\sigma} \mathrm{Mg}$ orbitals. The triangle-centered $\mathrm{s}$ orbitals are very weakly populated $(0.066$ at $3.20 \AA)$. The potential curve (see Figure 3) presents a minimum at $3.22 \AA$, but the RHF-I solution is not bound compared to the free atoms $\left(E_{\mathrm{coh} / \mathrm{at}}=-0.02 \mathrm{eV}\right)$. Let us note that this nonbinding result cannot be kept against the interstitial hypothesis since the triangular $\mathrm{Mg}$ lattice is only a convenient ideal system whose stability is not ensured.

The valence charge density map of the RHF-A solution is given in Figure 4b. The atomic character of this solution is obvious, the density maxima being located on circles centered on the $\mathrm{Mg}$ nuclei. The density minima are located at the centers of the triangles. The $\mathrm{Mg}-\mathrm{Mg}$ bond middle points correspond to saddle points. Moreover the analysis of the Mulliken populations indicates that more than $85 \%$ (1.705 at $3.90 \AA)$ of the population is on the $\mathrm{s} \mathrm{Mg}$ orbital, the missing electronic population being in the $\mathrm{p}_{\sigma}$ orbitals while the $\mathrm{s}$ triangle-centered orbitals are nearly empty. This solution is repulsive (see Figure 3 ) and dissociates well, toward RHF solution of the free atoms.

Let us now have a look at the band structures of these two RHF solutions. Figure 5 displays the two band diagrams along the ГМКГ path. The RHF-I one is typical of a semiconductor. In fact it is very similar to the $\pi$-band diagram of a graphite (001) slab, with a band crossing at the K point. The band width is equal to $6 t$, as for the graphite $\pi$ band, at the equilibrium distance $t=4.26 \mathrm{eV}$. On the other hand, the band diagram of RHF-A (Figure 5b) confirms its insulator closed-shell (filled band) character, with a large gap at the Fermi level $(3.1 \mathrm{eV})$.

All the above results (Mulliken populations, charge density map, band diagram) converge to the interpretation of the crossing point between the two RHF solutions as a first-order semiconductor-insulator phase transition. It would be very interesting to see the effects of the electronic correlation on this hypothesis. However, due to the very different nature of the two RHF solutions, one can reasonably expect that the inclusion of the correlation would not change the qualitative nature of the problem.

This system presents also a UHF antiferromagnetic interstitial solution (UHF-AF-I), as can be expected when a symmetry breaking occurs on an alternant lattice (let us remind that the inverse hexagonal, half-filled band lattice is a nonfrustated one and can support a Neel state). However the spin polarization is very weak, the energy stabilization being only $0.06 \mathrm{eV}$ (compared to the RHF-I solution), and the $\alpha-\beta$ population is 

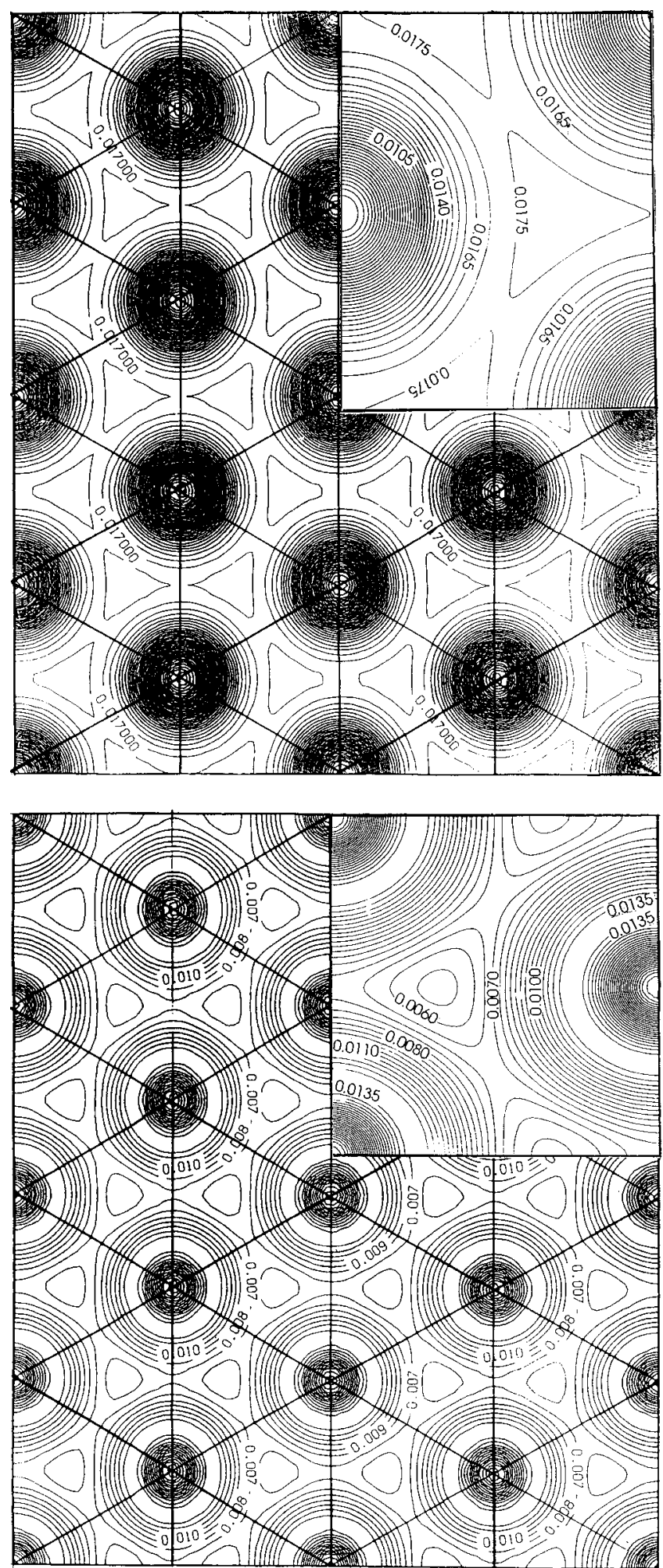

Figure 4. Magnesium triangular lattice. Valence charge density map of for the RHF solutions. The nuclei are located on the lattice vertices. (a, top) The interstice-centered one (3.2 ̊). (b, bottom) The atomcentered one $(3.9 \AA)$.

nonvanishing in the interstices only, and

$$
\frac{\rho_{\alpha}-\rho_{\beta}}{\rho_{\alpha}+\rho_{\beta}}=0.36
$$

From those results, one can see that the $\alpha$ and $\beta$ intersticecentered Wannier orbitals would have very large delocalization tails on the neighboring triangles; the overlap between nearest-
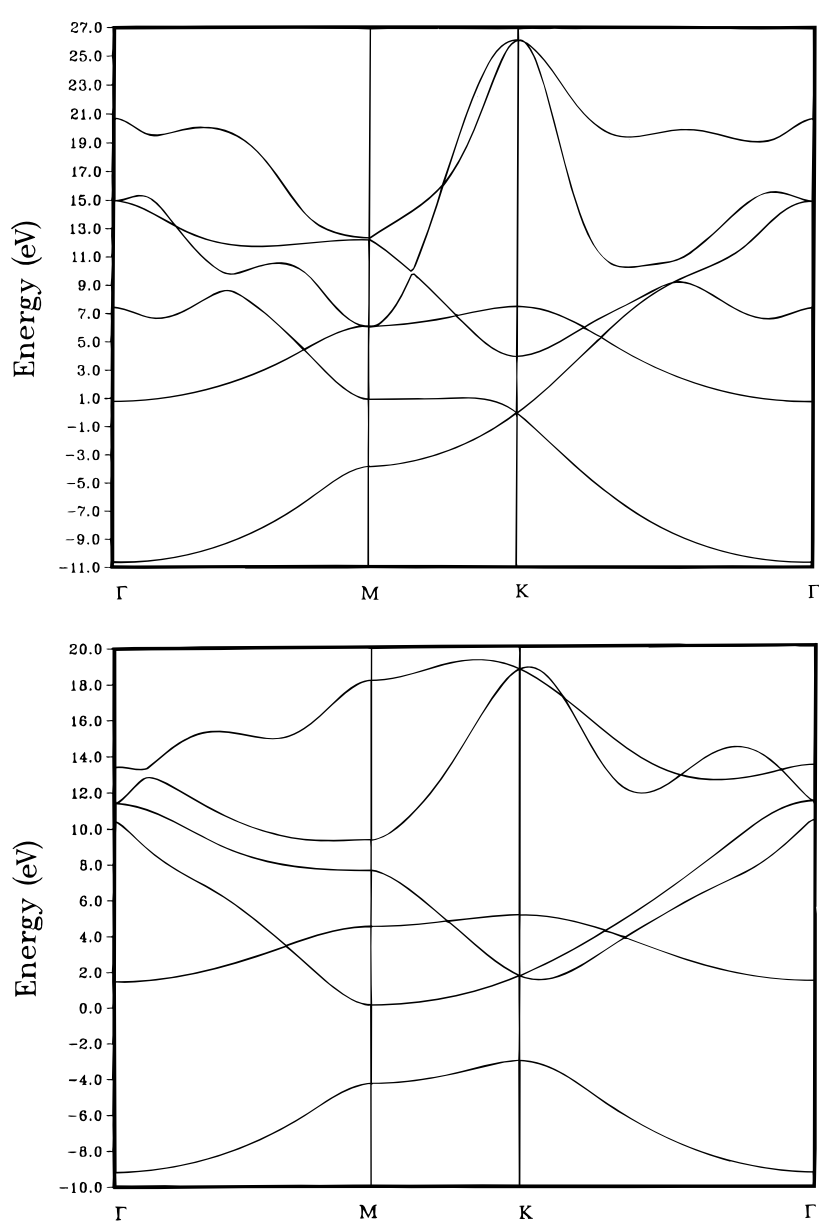

Figure 5. Magnesium triangular lattice. Band diagram of the RHF solutions. (a, top) The interstice-centered one (3.2 $\AA$ ). (b, bottom) The atom-centered one (3.9 ̊).

neighbor ones being close to 1 . This solution is slightly bound compared to the free atoms $\left(E_{\mathrm{coh} / \mathrm{at}}=0.05 \mathrm{eV}\right)$ at $R_{\mathrm{eq}}=3.23$ $\AA$ ).

The above results confirm the interstitial localization of the valence electrons, i.e., the first hypothesis of the interstitial/ magnetic model. However, the UHF interstitial solution is quasi-degenerated with the RHF-I one, its spin polarization is very small and its valence density (as for RHF-I) is delocalized over the whole inverse hexagonal lattice. All these reasons consolidate in order to weaken the systematic validity of the magnetic picture of the inverse lattice (points 2 and 3 of the model). The inverse "graphitic lattice" remains a highly delocalized system.

\section{Discussion}

The conclusion of the previous section confirms the validity of the description of simple metal systems in terms of IO. However, the up to now associated strongly correlated model has been proven wrong on the magnesium case. One can wonder why the lithium inverse lattice is relevant of a magnetic description while the magnesium one is relevant of a strongly delocalized picture. Different reasons can be given in order to explain this phenomenon.

(i) Let us look at the valence electronic densities per $\AA^{2}$. In the lithium Heisenberg solution, one finds $0.115 \bar{e} / \AA^{2}$, while in the RHF-I solution of the magnesium lattice one has $0.226 \bar{e} / \AA^{2}$, i.e., a density double than for the lithium square lattice. A magnetic picture supposes that each electron occupies a different orbital in an orthogonal set. A double density per 
$\AA^{2}$ therefore means orbitals twice as small and a kinetic energy twice as large. It is therefore understandable that it could be energetically more interesting to delocalize the electrons over the whole inverse lattice.

(ii) Another point of view is the energetic cost of the hybridization necessary to build nearly orthogonal $\alpha$ and $\beta$ sets of interstitial orbitals. While this condition is easy to get from an $s-p$ hybridization in the case of a square lattice (only four orbitals involved for each vertex), it is impossible without the participation of $\mathrm{d}$ orbitals for the triangular lattice (six orbitals involved at each vertex). Since d orbitals are very costly in terms of kinetic energy, it is more interesting to delocalize the electrons over the whole system.

The other point that needs to be discussed is the problem of the conductivity in a magnetic model, i.e, a model describing usually insulator systems. Let us first note that because of its strongly correlated nature, the lithium square system (in its inverse interstitial description) cannot be represented by a single determinant. The lowest reasonable approximation of its wave function is the ground-state solution of the Heisenberg Hamiltonian ( $\left.\Psi_{\text {Heis }}\right)$. The conductivity properties are therefore commanded by its ionization spectrum. As Malrieu mentioned in ref 12 , we shall show that the interaction matrix between the ionic states built from the Heisenberg ground state is isomorphic to a Hückel Hamiltonian. The relevant Hamiltonian for the singly ionic states is of course the $t-J$ Hamiltonian, while their vector space is generated by the $a_{p} \Psi_{\text {Heis, }} p$ referring to a local interstitial orbital, $a_{p}$ being the annihilation operator. One has

$$
\Psi_{\mathrm{Heis}}=\sum_{i} c_{i} \Phi_{i} \quad \text { with } \quad \sum_{i} c_{i}^{2}=1
$$

where $\Phi_{i}$ stands for a single determinant. Since half of the $a_{p} \Phi_{i}$ are zero, the ionic states should be renormalized to

$$
\Psi_{p}=\sqrt{2} a_{p} \Psi_{\text {Heis }}
$$

Let us now evaluate the interaction matrix:

$$
\begin{aligned}
&\left\langle\Psi_{p}\left|H_{t-J}\right| \Psi_{q}\right\rangle=\left\langle\Psi_{p}\left|H_{t}\right| \Psi_{q}\right\rangle-\left\langle\Psi_{p}\left|H_{J}\right| \Psi_{q}\right\rangle \\
&\left\langle\Psi_{p}\left|H_{J}\right| \Psi_{q}\right\rangle=2 \sum_{i} \sum_{j} c_{i} c_{j}\left\langle a_{p} \Phi_{i}\left|H_{J}\right| a_{q} \Phi_{j}\right\rangle \\
&=-2 \delta_{p q}\left(E_{\mathrm{Heis}}\left\langle\Psi_{\text {Heis }} \mid a_{p}^{\dagger} a_{p} \Psi_{\text {Heis }}\right\rangle+\right. \\
&\left.\sum_{k / k, p\rangle}\left\langle\Psi_{\text {Heis }} \mid a_{k}^{\dagger} a_{\bar{p}}^{\dagger} a_{\bar{k}} a_{p} \Psi_{\mathrm{Heis}}\right\rangle\right) \\
&=-\delta_{p q}\left(E_{\mathrm{Heis}}+\Delta E\right)
\end{aligned}
$$

where $\langle k, p\rangle$ means that $k$ and $p$ are nearest neighbors.

$$
\begin{aligned}
\left\langle\Psi_{p}\left|H_{t}\right| \Psi_{q}\right\rangle & =2 \sum_{i} \sum_{j} c_{i} c_{j}\left\langle a_{p} \Phi_{i}\left|H_{t}\right| a_{q} \Phi_{j}\right\rangle \\
& =2 \sum_{i} \sum_{j} c_{i} c_{j} t \delta_{\langle p, q\rangle} \delta_{i j} \\
& =t \delta_{\langle p, q\rangle}
\end{aligned}
$$

$\delta_{\langle p, q\rangle}=0$ if $p$ and $q$ are not nearest neighbors. Therefore, one sees that the ionic spectrum of the Heisenberg ground state is given by a matrix isomorphic to the Hückel one on the inverse lattice. The one-site energy is $E_{\text {Heis }}+\Delta E+U$ ( $U$ being the ionization energy) and the nearest-neighbor hopping integral is the one between two neighboring interstices. One has then recovered the familar ground of the usual band description.

\section{Conclusion}

Hartree-Fock calculations have been performed on 2-dimensional simple metal lattices: the square lithium lattice and the triangular magnesium lattice. The results obtained on both systems confirm the validity of the description of simple metals in terms of interstitial orbitals. The valence density of the lowest Hartree-Fock solution is always centered in the interstice between the atoms (squares or triangles). However, the original model was always associating interstitial electronic localization with a strongly correlated description in the inverse lattice. We have shown that those two ideas should be dissociated, since both strongly correlated (lithium lattice) and strongly delocalized (magnesium lattice) cases have been encountered. Their mutual relevance have been shown to be linked with the electronic density per unit space and the facility of building nearly orthogonal $\alpha$ and $\beta$ orbitals sets (in the antiferromagnetic interstitial UHF solution). The conductivity problem in a magnetic description has been investigated. It has been shown that the ionization spectrum of the ground state of a Heisenberg Hamiltonian is relevant of a description isomorphic to the classical band model.

Acknowledgment. We would like to thank C. Larrieu and M. Chaillet for the general help provided during this work. This work was partly supported by the EEC contract No. CHRXCT93-A55.

\section{References and Notes}

(1) McAdon, M. H.; Goddard, W. A. Phys. Rev. Lett. 1985, 55, 2563.

(2) McAdon, M. H.; Goddard, W. A. J. Phys. Chem. 1987, 57, 2607.

(3) Boustani, I.; Pewestorf, W.; Fantucci, P.; Bonacic-Koutecky, V.; Koutecky, J. Phys. Rev. B 1987, 35, 9437.

(4) Lepetit, M. B.; Malrieu, J. P.; Spiegelmann, F. Phys. Rev. B 1990, $41,8093$.

(5) Lepetit, M. B.; Aprà, E.; Malrieu, J. P.; Dovesi, R. Phys. Rev. B 1992, 46, 12974.

(6) Pople, J. A.; Nesbet, R. K. J. Chem. Phys. 1954, 22, 571.

(7) Dovesi, R.; Roetti, C.; Saunders, V. R. CRYSTAL92 User Manual; Universitá di Torino and SERC Daresbury Laboratory, 1992.

(8) Durand, P.; Barthelat, J. C. Gazz. Chim. Ital. 1978, 108, 205. 14,99

(9) Anderson, P. W. Phys. Rev. 1959, 115, 1; Solid State Phys. 1963

(10) Manousakis, E. Rev. Mod. Phys. 1991, 63, 1 and references therein.

(11) Barriol, J.; Metzer, J. J. Chim. Phys. 1950, 47, 432.

(12) Malrieu, J. P.; Maynau, D.; Daudey, J. P. Phys. Rev. B. 1984, 30, 1817.

JP953119G 